\title{
Ways to Knowledge-First Believe
}

\section{Simon Wimmer ${ }^{1}$ (1)}

Received: 1 January 2020 / Accepted: 9 March 2021 / Published online: 28 May 2021

(c) The Author(s) 2021

\section{Abstract}

On a widely suggested knowledge-first account of belief, to believe $p$ is to $\Phi$ (e.g. treat $p$ ) as if one knew $p$. I challenge this view by arguing against various regimentations of it. I conclude by generalizing my argument to alternative knowledge-first views suggested by Williamson and Wimmer.

\section{Introduction}

What is it to believe $p$ ? How are knowing $p$ and believing $p$ related? To answer both questions at once, we may characterize believing $p$ by appeal to knowing $p$ and so put knowledge first. One way of doing this is to say that to believe $p$ is to $\Phi$ as if one knew $p .{ }^{1}$ Call this view AS IF. Variants of AS IF are widely suggested. Here are three representative quotes:

[...] we can define the belief that $p[\ldots]$ as the disposition to act (think, feel) as one would if one knew that $p$. (Hyman 2017, p. 284) ${ }^{2}$

Believing is $[\ldots]$ a shadow or after-effect of knowing: the deceived agent who reaches for the basket behaves as if she knew that the ball is in the basket. (Nagel 2017 , p. 537)

\footnotetext{
${ }^{1}$ I use ' $\Phi$ ' as a placeholder, rather than variable.

2 Hyman (2017, p. 285) suggests that, if we take knowledge not to entail belief, but only to be normally accompanied by it, "we can [instead] define the belief that $p$ as the disposition to act (think,feel) as one normally or generally would if one knew that $p$." Most of my discussion below generalizes to this view.

${ }^{3}$ Note that in the passage from which the quote is taken, Nagel is not concerned with giving an account of belief, but with saying what is involved in attributing belief.
}

$\bowtie \quad$ Simon Wimmer

simon.wimmer@tu-dortmund.de

1 Department of Philosophy and Political Science, TU Dortmund, Emil-Figge-Str. 50, 44227

Dortmund, Germany 
[...] believing $p$ is, roughly, treating $p$ as if one knew $p$ [...]. (Williamson 2000, p. 47$)^{4}$

My aim here is to develop a challenge for AS IF. I will argue against various possible regimentations of AS IF, including those suggested in the extant literature. Absent another more satisfactory regimentation, I suggest that we should not accept AS IF. For concreteness, I will substitute 'treat $p$ ' for ' $\Phi$ ' throughout. As is easily verified, however, my arguments mutatis mutandis go through also if we substitute 'behave' or 'is disposed to act (think, feel)' instead.

Section 2 introduces an initial regimentation of AS IF which, following Wimmer (2019), appeals to an existential quantifier over ways whose domain is restricted by context. Section 3 argues against this regimentation and views in its vicinity which retain a contextually determined domain restriction on their quantifier over ways. Section 4 casts doubt on not imposing a domain restriction at all. Section 5 argues against imposing a domain restriction via an explicit clause in the theory. Finally, Sect. 6 concludes and sketches how my discussion generalizes to previous regimentations and refinements of AS IF suggested by Williamson (2000, pp. 46-7) and Wimmer (2019).

A comment about my strategy before I get started. Because I focus on regimentations of AS IF, defenders of the view may reply by insisting that unregimented AS IF makes the right predictions, even if the regimentations do not. ${ }^{5}$ However, this reply is unsatisfactory. For there seem to be clear cases of belief, where the view, if left unregimented, does not deliver clear predictions.

To illustrate, consider a burglar who relies on the proposition that the diamond is in Jess' house (' $d$ ') as a premise in planning her nightly route. As she does this not just because she takes $d$ to sufficiently approximate the truth, it is natural to say that she believes $d$. However, the burglar does not know $d$, since her belief is based on the false lemma that the diamond is under the bed. ${ }^{6}$ Given this fact about basing, she treats $d$ as defeasible by evidence that the diamond is not under the bed. But this is quite unlike how the burglar would treat $d$ if she knew it. For if she knew $d$, her believing $d$ would not be based on the false lemma that the diamond is under the bed; and she would

\footnotetext{
${ }^{4}$ Holton (2017) and Nagel (2013) seem to endorse this suggestion. Williamson, however, only endorses a refinement of this suggestion, which I discuss briefly in my conclusion. See Roessler (2013, pp. 323-4) for brief critical discussion of both the suggestion in the text and Williamson's refinement.

5 As an anonymous reviewer noted, defenders of AS IF may try to motivate this insistence by noting that 'believe' is a vague term. Given this, they may say, we should expect that the precise regimentations I focus on are extensionally inadequate, as this is the result we get if we attempt to impose precision on phenomena picked out by vague expressions. However, although I am happy to concede that 'believe' is a vague term, it is unclear whether this motivates insisting on unregimented AS IF. For one, unregimented AS IF seems to predict indecision even in clear cases of belief, such as the case of the burglar in the following two paragraphs. This means that unregimented AS IF, although a vague theory of a vague entity, predicts vagueness where there is none. For another, the regimentations I discuss are not in fact fully precise. Many of them contain vague expressions themselves (e.g. 'contextually relevant'). But given this, regimentations of AS IF are, for all we know, consistent with the vagueness of 'believe'. Moreover, they may yet receive further support from it: for all we know, the regimentations even explain the vagueness of 'believe' in terms of the vagueness of some other expressions in our language (like 'contextually relevant').

${ }^{6}$ I here assume that it is metaphysically impossible to know $p$ if one believes $p$ based on a false lemma. This assumption is widely accepted, but not uncontroversial. See, e.g., Buford and Cloos (2018); Schnee (2015); Turri (2019); Warfield (2005) for discussion.
} 
not treat $d$ as defeasible by evidence that the diamond is not under the bed. ${ }^{7}$ So, it is natural to say, at least initially, that she does not treat $d$ as if she knew it. And yet, on further reflection, it is equally natural to say that she does treat $d$ as if she knew it. For the burglar relies on $d$ as a premise and would do so also if she knew $d$. Without saying more about treating as if one knew, it is now unclear how we should settle whether the burglar treats $d$ as if she knew it. But this means that without further regimenting AS IF, it remains unclear what predictions the view makes about the burglar. This provides reason to regiment AS IF.

\section{An Initial Regimentation}

How should we regiment AS IF? A natural starting point is Wimmer (2019)'s regimentation: ${ }^{8}$

$\exists$ AS IF For all $x$ and $p, x$ believes $p$ iff: for some contextually salient way $F$, (i) $x$ treats $p$ in $F$ and (ii) if $x$ knew $p, x$ would treat $p$ in $F$.

Wimmer motivates this regimentation via linguistic considerations. ${ }^{9}$ Notice, first, that the clause to the right of 'as', 'if one knew it,' is in the subjunctive mood. We may thus interpret it as the antecedent of a would-counterfactual whose consequent is elided. Recovering the elided material, AS IF says, roughly, that one believes $p$ iff one treats $p$ as one would treat it if one knew it. Wimmer then adopts a semantics of the comparative particle 'as' inspired by Bücking (2017)'s treatment of German 'wie'. On this semantics, 'as' denotes an equivalence relation between individuals or eventualities. This relation holds of two individuals or eventualities just in case they are the same in a certain contextually salient way. Applying this to AS IF, it says that one believes $p$ iff one treats $p$ in some contextually salient way $F$ and if one knew it, one would treat $p$ in $F$. Thus, we have $\exists$ AS IF.

Another motivation for regimenting AS IF via $\exists$ AS IF is that $\exists$ AS IF makes clear and correct predictions about the burglar, at least on the modest assumption that the burglar's reliance on $d$ as a premise is made contextually salient by the description of the case given above. For given this assumption, some contextually salient way $F$ is such that the burglar treats $d$ in $F$ and would treat $d$ in $F$ if she knew $d$. So, $\exists$ AS IF clearly and correctly predicts that the burglar believes $d$.

Given these two motivations, I take $\exists$ AS IF to be a natural starting point for our discussion. Before proceeding, however, three comments about $\exists$ AS IF and the other regimentations of AS IF I consider below are in order. First, all of them appeal to a would-counterfactual. This interpretative choice is plausible in light of the linguistic consideration Wimmer highlights, namely that the clause to the right of 'as' in AS IF, 'if

\footnotetext{
${ }^{7}$ Note that this difference between the mere belief and knowledge conditions is compatible with the burglar in the knowledge condition treating $d$ as defeasible by a wide range of (misleading) evidence. So, my description of the example does not require that knowledge is incompatible with certain kinds of defeat. 8 In line with my general policy, I have 'treats $p$ ' where Wimmer has ' $\Phi$ s.'

9 As Wimmer notes, this regimentation diverges from unregimented AS IF in one respect. The latter defines what it is to believe a proposition. This definition is not equivalent to the biconditional in $\exists$ AS IF. However, its predictions about cases are the same. Consequently, it suffers from the same counterexamples as $\exists$ AS IF.
} 
one knew it,' is in the subjunctive mood and so naturally interpreted as the antecedent of a would-counterfactual whose consequent is elided. However, my arguments against $\exists$ AS IF and the other regimentations of AS IF I consider are largely independent of this interpretative choice. Most go through also if we replace occurrences of wouldcounterfactuals with occurrences of normality conditionals ('normally, if $\phi, \psi$ ') or strict conditionals ('necessarily, if $\phi, \psi$ ') throughout.

Second, $\exists$ AS IF, like the other regimentations of AS IF I consider, appeals to ways. I will not say much about ways here, but I will assume a close link between ways and 'how'-questions: if a (partial) answer to the question 'How does $x \phi$ ?' cites $F$, then we have prima facie reason to think that $F$ is a way of $\phi$ ing (Breckenridge 2007). ${ }^{10}$ This assumption is corroborated by our explicit uses of the noun 'way' in English. To illustrate, suppose we ask how Jeff treats Jill. One (partial) answer to this question is 'He treats her poorly'. Note that, in response to this answer, we may felicitously utter 'that's no way to treat Jill!' or 'why does Jeff treat her that way?' This supports what the close link above predicts, namely that we have prima facie reason to think that treating Jill poorly is a way of treating Jill. For another example, note that another (partial) answer to our question is 'Jeff does not answer Jill's calls.' Here too we may felicitously respond by saying 'that's no way to treat Jill!' or 'why does Jeff treat her that way?' This again supports what the close link above predicts, namely that we have prima facie reason to think that not answering Jill's calls is a way of treating Jill. For a final example, which will feature in my argument below, suppose we ask how Joan (the meteorologist) treats the proposition that it is not raining (' $\neg r$ '). One (partial) answer to this question is 'Joan relies on $\neg r$ in her (meteorological) computations.' In response to this answer, we may then felicitously utter 'that's a strange way for her to treat $\neg r$; she doesn't believe it after all!' or 'why does Joan treat $\neg r$ that way?' Again, this confirms what the close link above predicts, namely that we have prima facie reason to think that relying on $\neg r$ in computations is a way of treating $\neg r$.

Finally, the clause ' $x$ treats $p$ in $F$ ' as it occurs in $\exists$ AS IF and the other regimentations of AS IF I consider is to be read as a habitual. Paradigm instances of habituals are sentences or clauses in simple present tense, e.g. 'John smokes' or 'Mary handles the mail from Antarctica.' These may be true at a world-time pair $\langle w, t\rangle$ even if no event of John smoking or of Mary handling the mail from Antarctica occurs at $\langle w, t\rangle$. The second may even be true if no such event has ever occurred. ${ }^{11}$ If we read the clause ' $x$ treats $p$ in $F$ ' as a habitual, one may satisfy $\exists$ AS IF's condition (i) at $\langle w, t\rangle$ even if one is not the agent of a treating event at $\langle w, t\rangle$ (so, for instance, even if one is in a dreamless sleep). ${ }^{12}$

\footnotetext{
${ }^{10}$ For discussion of the semantics of 'how'-questions and various distinctions between kinds of ways see Jaworski (2009); Sæbø (2016). See also Stanley and Williamson (2001); Bengson and Moffett (2011) for further discussion of ways of acting.

11 See Fara (2005); Boneh and Doron (2010, 2012); Ferreira (2016) for discussion of habituals.

12 The unregimented version of AS IF does not feature the clause ' $x$ treats $p$ in $F$.' However, it features the infinitival clause 'to treat $p$ as if one knew $p$ ' and this clause too is to be read as a habitual. Although paradigm habituals are in simple present tense, infinitival clauses also often receive a habitual interpretation. Consider Huddleston and Pullum (2002, p. 161)'s example: 'He seems to do 'The Times' crossword.' On a natural reading, this sentence may be true at a world-time pair $\langle w, t\rangle$ even if no event of him doing 'The Times' crossword seems to occur at $\langle w, t\rangle$.
} 


\section{The Initial Challenge}

This section starts to develop my challenge for AS IF by arguing against $\exists$ AS IF. My argument turns on the domain restriction on $\exists$ AS IF's existential quantifier to contextually salient ways. Depending on how we set up the context, this restriction is either too permissive or too restrictive.

The possibility of two kinds of contexts drives my argument against $\exists$ AS IF: silent and noisy ones. A context $c$ is silent iff no way $F$ such that one treats $p$ in $F$ and if one knew $p$, one would treat $p$ in $F$ is contextually salient in $c$. A context $c$ is noisy iff there is at least one way $F$ contextually salient in $c$ such that one treats $p$ in $F$ and if one knew $p$, one would treat $p$ in $F$, although one does not believe $p$.

The challenge silent and noisy contexts pose for $\exists$ AS IF is simple. In silent contexts, the domain restriction on $\exists$ AS IF's existential quantifier is so restrictive as to guarantee that no way $F$ satisfies conditions (i) and (ii). Even if one believes $p$, one does not satisfy $\exists$ AS IF's right-hand-side and is predicted not to believe $p$. Thus, $\exists$ AS IF is subject to counterexamples.

In noisy contexts, the domain restriction on $\exists$ AS IF's existential quantifier is so permissive as to guarantee that some way $F$ satisfies conditions (i) and (ii). Although one does not believe $p$, one thus satisfies $\exists$ AS IF's right-hand-side and is predicted to believe $p$. Thus, $\exists$ AS IF faces yet more counterexamples.

My argument against $\exists$ AS IF so far is rather abstract. It depends only on the claim that silent and noisy contexts are possible; thus, it is independent of specific claims about what contexts are and how they determine which ways are contextually salient as long as those claims allow for the possibility of silent and noisy contexts. For concreteness though, the next subsection illustrates how my argument proceeds given a specific framework for thinking about contexts.

\subsection{Illustrating the Challenge}

Suppose we model a context as including a question under discussion (henceforth 'QUD'). ${ }^{13}$ The QUD may correspond to an earlier question asked by a conversational participant or it may have been indirectly raised as the conversation evolved. Contextual salience (henceforth 'salience') is now defined via the QUD. A conversational move $m$ is salient to the QUD $Q$ iff $m$ either expresses a (partial) answer to $Q$ ( $m$ is an assertion) or is part of a strategy to answer $q$ ( $m$ is a question). This allows us to define salience for an entity, e.g. a location or way: An entity $e$ is salient in a context $c$ with QUD $Q$ iff either $e$ is cited by a (partial) answer to $Q$ or $e$ is cited by part of a strategy to answer $Q{ }^{14}$

To illustrate my challenge to $\exists$ AS IF, first consider a case involving a silent context. Suppose Ayesha believes that it is raining (' $r$ '). She asks Delia where John is. The

\footnotetext{
13 The model of context and contextual salience I use here is developed in Roberts (2012), although she uses 'relevance' where I use 'salience'. See Elswyk (2019, section 2) for a concise summary.

14 Because I do not have a definition of what it is for an entity to be cited by a (partial) answer to, or part of a strategy to answer, $Q$, this definition is vague. But for present purposes, nothing hangs on how that vagueness is resolved.
} 
QUD is now where John is. This makes salient the locations at which John may be located since these may be cited in a (partial) answer to the QUD. No way of treating $r$ is salient, as no question about such ways has been asked or indirectly raised as the conversation evolved. So, no way $F$ such that Ayesha treats $r$ in $F$ and if she knew $r$, she would treat $r$ in $F$ is contextually salient. So, in this context, $\exists$ AS IF mistakenly predicts that Ayesha does not believe $r{ }^{15}$

Second, consider a case involving a noisy context. Suppose Joan does not believe $\neg r$. Joan is a meteorologist, who is modelling certain weather patterns. She is interested in the weather conditional on $\neg r$ and would be interested in this even if she knew $\neg r$. So, she uses $\neg r$ as a premise in her computations and would do so also if she knew $\neg r$. Delia now asks whether Joan uses $\neg r$ as a premise in her computations. Delia's question is the QUD and makes using $\neg r$ as a premise in modelling certain weather patterns salient. So, some contextually salient way $F$ is such that Jess treats $\neg r$ in $F$ and she would treat $\neg r$ in $F$ if she knew $\neg r$. So, in this context, $\exists$ AS IF mistakenly predicts that Joan believes $\neg r{ }^{16}$

15 We might reply to this argument by amending the right side of the biconditional in $\exists$ AS IF to feature a conditional. However, this reply faces some difficulties of its own. Suppose, for starters, we use a material conditional:

- For all $x$ and $p, x$ believes $p$ iff: for some way $F$, if $F$ is contextually salient, then (i) $x$ treats $p$ in $F$ and (ii) if $x$ knew $p, x$ would treat $p$ in $F$.

Here, the right-hand side is satisfied if for some way $F$, it is not the case that $F$ is contextually salient. Consequently, silent contexts remain problematic. Put roughly, since, in silent contexts, there are many ways that are not contextually salient, $\exists$ AS IF now predicts that any subject believes any proposition, whether or not they actually do.

Alternatively, we may, as an anonymous reviewer suggests, use a counterfactual conditional if $F$ were contextually salient, then (i) and (ii) would be the case.' However, this option too is subject to counterexample. Very roughly, the problem is that bringing a way of treating to salience may affect whether one treats a given proposition in that way. This results in the possibility of cases where one does not believe $p$, yet satisfies $\exists$ AS IF's right-hand side.

To illustrate, consider Dilek who has never considered the proposition that $177+23=200$ and does not believe it, although she is disposed to do so. (See Audi (1994) for relevant discussion of the distinction between (dispositional) beliefs and dispositions to believe, and why cases like Dilek's involve dispositions to believe, rather than (dispositional) beliefs.) Suppose Dilek is discussing some maths problems with her friend Alex, and that she is in a silent context. Now, what would happen if, for instance, relying on the proposition that $177+23=200$ were salient? Given how things are at the actual world (Alex and Dilek discussing maths), that way would likely be salient because Alex asks Dilek whether she relies on the proposition at issue or does something similar which brings the proposition to Dilek's attention. But once Dilek would attend to the proposition that $177+23=200$, she would come to believe it (she has pretty good mathematical cognition at the actual world) and to rely on it in her reasoning. Moreover, it would also be the case that if Dilek knew the proposition at issue, she would rely on it in her reasoning. So, in the present case, if relying on the proposition that $177+23=200$ were salient, Dilek would satisfy conditions (i) and (ii). The amended version of $\exists$ AS IF predicts her to believe that $177+23=200$, despite the fact that she does not.

Clearly, more can be said about examples of this kind. But, one lesson we may draw nonetheless is that appeal to a conditional, material or counterfactual, does not straightforwardly help defenders of AS IF. (As far as I can see, this holds also for other types of conditional, such as the normality and strict conditional.)

16 Defenders of $\exists$ AS IF may reply to this case by denying that using $\neg r$ as a premise in her computations is a way in which Joan treats $\neg r$. However, as noted in Sect. 2, we have prima facie reason to regard using $\neg r$ as a premise in her computations as a way in which Joan treats $\neg r$. Of course, as inquiry progresses, this prima facie reason may be defeated. But on general methodological grounds, we should treat it as such only if we have reason to do so. The burden is thus on defenders of $\exists$ AS IF to provide a reason for doing 
Ayesha's and Joan's cases illustrate my challenge to $\exists$ AS IF. Due to silent and noisy contexts, $\exists$ AS IF is subject to counterexamples. I now generalize this challenge to other views in $\exists$ AS IF's vicinity.

\subsection{Generalizing the Challenge}

Say we substitute $\exists$ AS IF's existential quantifier with the definite determiner 'the':

$\iota$ AS IF For all $x$ and $p, x$ believes $p$ iff: the contextually salient way $F$ such that (i) $x$ treats $p$ in $F$ is also such that (ii) if $x$ knew $p, x$ would treat $p$ in $F$.

'The $X$ is $Y$ ' is usually thought to be true only if there is some unique element in the domain that satisfies the (possibly complex) predicate $X$ or to presuppose that there is such an element. (Hawthorne and Manley 2012) If there is no element that satisfies the predicate, we thus get either falsity or a truth-value gap. Consequently, in the above silent context, Ayesha is predicted not to believe $r$. Moreover, the noisy context above can easily be set up so that there is a unique salient way $F$ in which Joan treats $\neg r$ and which is also such that if Joan knew $\neg r$, she would treat $\neg r$ in $F$. So, in that context, ८ AS IF mistakenly predicts that Joan believes $\neg r$.

Defenders of AS IF may be happy to concede that $\iota$ AS IF must be rejected. For it fares even worse than $\exists$ AS IF in loquacious contexts where there is no unique way $F$ in which one treats $p$ which is contextually salient. In a loquacious context, $\iota$ AS IF yet again faces counterexamples.

But, substituting 'most', for instance, yields mistaken predictions as well.

$\mu$ AS IF For all $x$ and $p, x$ believes $p$ iff: most contextually salient ways $F$ such that (i) $x$ treats $p$ in $F$ are also such that (ii) if $x$ knew $p, x$ would treat $p$ in $F$.

'Most $X \mathrm{~s}$ are $Y$ ' is usually thought to be true iff the number of $X \mathrm{~s}$ that are $Y$ is strictly greater than the number of $X$ s that are not $Y$ (Barwise and Cooper 1981). ${ }^{17}$ Since, in the silent context above, there is no salient way of treating a proposition, the number of contextually salient ways $F$ such that Ayesha treats $r$ in $F$ is 0 . So, the number of such ways that are also such that if Ayesha knew $r$, she would treat $r$ in them equals the number of such ways that are not such that if Ayesha knew $r$, she would treat $r$ in them. Both are 0. So, Ayesha is mistakenly predicted not to believe $r$.

This result is avoided if we substitute the universal quantifier for $\exists$ AS IF's existential quantifier:

$\forall$ AS IF For all $x$ and $p, x$ believes $p$ iff: every contextually salient way $F$ such that (i) $x$ treats $p$ in $F$ is also such that (ii) if $x$ knew $p, x$ would treat $p$ in $F$.

If the domain of a universal quantifier that heads a universally quantified sentence ' $\forall x \phi(x)$ ' is empty, that sentence is vacuously true. So, in the earlier silent context, where there is no contextually salient way $F$ such that Ayesha treats $r$ in $F$, she

so. Moreover, at the beginning of Sect. 4 I give a few more examples that could be used in place of Joan's here. This adds to the burden on defenders of $\exists$ AS IF, as they must show that these examples too rely on what are in fact not ways of treating in order to avoid all counterexamples.

17 That is, 'Most $X \mathrm{~s}$ are $Y \mathrm{~s}$ ' is true iff $|X \cap Y|>|X-Y|$. 
vacuously satisfies $\forall$ AS IF's right hand side. Thus, $\forall$ AS IF predicts Ayesha to believe $r$.

However, although $\forall$ AS IF does not undergenerate in silent contexts, it does overgenerate in them. Suppose Ayesha does not believe that it is not raining (' $\neg r$ '). She asks Delia where John is. The QUD is now where John is. This makes salient the locations at which John may be located. But, as no question about ways of treating $r$ has been asked or indirectly raised as the conversation evolved, no way of treating $r$ is salient. So, no way $F$ such that Ayesha treats $\neg r$ in $F$ and if she knew $\neg r$, she would treat $\neg r$ in $F$ is salient. Thus, $\forall$ AS IF mistakenly predicts that Ayesha believes $\neg r$.

Overgeneration in silent contexts is not the only problem for $\forall$ AS IF. Overgeneration in noisy contexts is another one, which affects $\mu$ AS IF as well. The above noisy context involving Joan and Delia can easily be set up so that the unique salient way in which Joan treats $\neg r$ is also such that if Joan knew $\neg r$, she would treat $\neg r$ in it. Consequently, most/all contextually salient ways $F$ such that Joan treats $\neg r$ in $F$ are also such that if she knew $\neg r$, she would treat $\neg r$ in $F$. So, in this context, Joan is mistakenly predicted to believe $\neg r$ by both $\mu$ AS IF and $\forall$ AS IF.

This subsection generalized my challenge to $\exists$ AS IF to some views in $\exists$ AS IF's vicinity. These views varied the type of quantifier over ways, but held fixed appeal to context to determine a domain restriction on said quantifier. I now turn to views that reply to my challenge by abandoning appeal to context and imposing no domain restriction. These unrestricted views avoid the challenge silent and noisy contexts pose. But absent an alternative domain restriction they are nonetheless problematic.

\section{No Restriction}

Unrestricted $\exists$ AS IF overgenerates. Joan uses $\neg r$ as a premise in her computations and would do so also if she knew $\neg r$. Consider also Dan who is doing logic exercises and, although he does not believe that it is raining, treats $r$ as a premise whilst doing a conditional proof of if $r$, then $r$. If he knew $r$, he would still do so. Finally, consider Irene who has cultivated a disposition to be extremely careful in surveying the logical and evidential relations between propositions. She does not believe that it is raining, yet treats that proposition with great care. Given her disposition for extreme care, she would treat $r$ with great care also if she knew it. Unrestricted $\exists$ AS IF mistakenly predicts Joan to believe $\neg r$ and Dan as well as Irene to believe $r$.

Unrestricted $\iota$ AS IF undergenerates. This is due to uniqueness failure. There are many ways in which Ayesha treats $r$. For instance, she uses it as a premise in her reasoning, treats it as a reason to bring an umbrella, etc. Consequently, there is no unique way in which Ayesha treats $r$. But, as I mentioned earlier, 'the $X$ is $Y$ ' is usually thought to be true only if there is some unique element in the domain that satisfies the (possibly complex) predicate $X$ or to presuppose that there is such an element. Thus, unrestricted $\iota$ AS IF mistakenly fails to predict that Ayesha believes $r$.

Unrestricted $\forall$ AS IF also undergenerates. This is because moving from merely believing to knowing $p$ often makes a difference to how one treats $p$. Consider a case involving a burglar, inspired by Williamson (2000). This burglar merely believes that the diamond is in Jess' house (' $d$ ') because her believing $d$ is based on the false lemma 
that the diamond is under the bed. ${ }^{18}$ Because of this fact about basing, the burglar treats $d$ as defeasible by evidence that the diamond is not under the bed. But if she knew $d$, she would not believe $d$ based on a false lemma and so would not treat $d$ as defeasible by evidence that the diamond is not under the bed.

Unrestricted $\forall$ AS IF mistakenly predicts that the burglar does not believe $d$. For the burglar treats $d$ in ways $F_{1}-F_{n}$. But if she knew $d$, at least one of $F_{1}-F_{n}$, call it $F_{i}$, would be such that the burglar would not treat $d$ in $F_{i}$. So, the burglar does not satisfy unrestricted $\forall$ AS IF's right hand side. ${ }^{19}$

Cases like the burglar's may not be counterexamples to unrestricted $\mu$ AS IF. Although not every way in which the burglar treats $d$ is such that she would treat $d$ in that way if she knew $d$, defenders of AS IF may say that most ways in which the burglar treats $d$ are. If so, unrestricted $\mu$ AS IF predicts the burglar to believe $d$.

However, even unrestricted $\mu$ AS IF faces a challenge absent a restriction on its quantifier over ways. Consider Ross, who is a logician. Although he does not believe it, he is interested in the inferential potential of the proposition that the moon is made of cheddar (' $m$ '). Being a classical logician, Ross treats $m$ as entailing $m \vee q$ for any proposition $q$. Since there are infinitely many propositions, there are infinitely many ways in which he treats $m$-call them $F_{1}-F_{\infty}$. Moreover, we can suppose that even if Ross knew $m$, he would be interested in its inferential potential and so would treat $m$ in $F_{1}-F_{\infty}$. This means that the number of ways in which Ross treats $m$ which satisfy conditions (i) and (ii) of $\mu$ AS IF is infinitely large.

To evaluate whether unrestricted $\mu$ AS IF predicts Ross to believe $m$, we need to consider the number of ways which satisfy condition (i), but not condition (ii), of unrestricted $\mu$ AS IF. Unrestricted $\mu$ AS IF mistakenly predicts Ross to believe $m$ if that number is finite, infinite but smaller than the number of ways in which Ross treats $m$ which satisfy conditions (i) and (ii), or infinite but equal to the latter number. The challenge for defenders of unrestricted $\mu$ AS IF is now to show that the number of ways which satisfy condition (i), but not condition (ii), of unrestricted $\mu$ AS IF is none of these. Without a response to this challenge on hand, we should not accept unrestricted $\mu$ AS IF. ${ }^{20}$

\footnotetext{
18 I here again assume that it is metaphysically impossible to know $p$ if one believes $p$ based on a false lemma. See fn.6 for references relevant to this assumption.

19 Another counterexample to unrestricted $\forall$ AS IF is a rock, or some other inanimate object. Such an object does not treat a person, proposition or other entity in any way. So, there is no way $F$ such that the rock treats $p$ in $F$. So, the rock vacuously satisfies $\forall$ AS IF's right hand side. Since it does for any $p$, the rock is predicted to believe any proposition.

In reply, defenders of unrestricted $\forall$ AS IF may restrict the range of values $x$ may take to animate subjects only. This rules out the rock as a potential believer. However, depending on how animacy is understood, it also rules out groups and artificial entities as believers. Since it is often thought that groups and artificial entities can have beliefs, defenders of AS IF owe us either an explanation of why they in fact cannot or a suitable account of animacy. Alternatively, they could restrict the range of values $x$ may take to minded subjects. This may do better than a restriction to animate subjects since groups and artificial entities may be minded even if inanimate.

20 A similar challenge also arises for views that replace the generalized quantifier 'most' with a contextsensitive proportional quantifier 'sufficiently many.' The resultant views, however, also face the additional challenge of saying how context determines the threshold of the proportional quantifier. Responding to this added challenge is further complicated by the threat of contexts similar to silent ones which do not
} 


\section{Theory-Driven Restriction}

So far, I have considered regimentations of AS IF which either do not appeal to a domain restriction on their quantifier over ways or appeal to a contextually determined restriction. But defenders of AS IF do not have to throw out appeal to a domain restriction just because they throw out appeal to a contextually determined domain restriction. They may also impose a theory-driven domain restriction; a restriction given via an explicit clause in their theory. I now argue against this option.

We can divide theory-driven restrictions into two camps. Defenders of AS IF may restrict their quantifier's domain either (i) to a fully specific way of treating $p$, or (ii) to a less than fully specific way of treating $p$ or some subset of such ways. To introduce the distinction between fully and less then fully specific ways of treating $p$, recall that I closely linked ways and 'how'-questions. I say that a way of $\phi \mathrm{ing}, F$, is fully specific iff a complete answer to the question 'How does $x \phi$ ?' cites $F$. By contrast, $F$ is less than fully specific iff it is not fully specific or, equivalently, iff a merely partial answer to the question 'How does $x \phi$ ?' cites $F$.

Examples of complete and merely partial answers help to distinguish fully and less than fully specific ways:

1. Suppose all and only Sue, Mary, Bill, and John are coming to the party.

(a) Who is coming to the party?

(b) Sue, Mary, Bill, and John are coming.

(c) Sue is coming.

complete answer merely partial answer

Here, the complete answer lists all people coming to the party. The merely partial answer, by contrast, lists only some of them.

Analogously, a complete answer to the question 'How does $x \phi$ ?' lists all ways in which $x \phi$ s. The fully specific way in which $x \phi$ s is then the conjunction or composite of all these ways, whereas these ways themselves and any conjunctions or composites formed from them that are distinct from the fully specific way in which $x \phi$ s are less than fully specific ways. ${ }^{21}$

Suppose defenders of AS IF restrict the domain of their quantifier over ways to some less than fully specific ways of treating $p$. There are many candidates. Using $p$ as a premise is one. Asserting $p$ is another. Whichever candidate or subset of the candidates defenders of AS IF choose, they are in an uncomfortable position.

Suppose they choose using $p$ as a premise. Why choose this, rather than some other less than fully specific way of treating $p$ or subset of such ways? Presumably, because this choice ensures AS IF's extensional adequacy, i.e. that one believes $p$, according to AS IF, iff one in fact believes $p$. But if so, their appeal to knowledge in clause (ii) of AS IF is an idle wheel, at least in securing its extensional adequacy: The view that one believes $p$ iff one uses $p$ as a premise makes the same predictions as the proposed

\footnotetext{
determine a threshold and contexts similar to noisy ones which determine a threshold that is too permissive. I leave developing this added challenge for another occasion.

21 This uses less than fully specific ways to understand what fully specific ways are. Another option is to understand less than fully specific ways in terms of fully specific ways. On this view, a less than fully specific way may be defined as a set of fully specific ways. Using $p$ as a premise, for instance, is then the set of fully specific ways of treating $p$ such that a subject who treats $p$ in that way uses $p$ as premise.
} 
regimentation of AS $\mathrm{IF}^{22}$ Yet this view is simpler and so, other things being equal, preferable to the proposed regimentation of AS $\mathrm{IF}^{23}$

The point here is general. It holds for any candidate less than fully specific way of treating $p$ or subset of such candidates. For any of these, we get a view of belief that is extensionally equivalent to the proposed regimentation of AS IF that appeals to them, but preferable to it on grounds of simplicity.

The second option for defenders of AS IF, restricting their quantifier's domain to fully specific ways of treating $p$, avoids this concern. Unlike a restriction to less than fully specific ways, this restriction does not filter out any irrelevant details. The theory leaves that task to appeal to knowledge.

In the special case of subjects that treat $p$ in some ways, the restriction to fully specific ways of treating $p$ collapses differences between the variants of AS IF I considered earlier. Because for any such subject $x$ and proposition $p$ there is some unique fully specific way in which $x$ treats $p$, the domain of whatever quantifier we choose is now guaranteed to be non-empty and to contain no more than one element. Given this, it makes no difference whether we use the definite determiner 'the,' generalized quantifier 'most,' existential, or universal quantifier. Whichever way we go, AS IF now predicts that one believes $p$ iff one's fully specific way $F$ of treating $p$ is such that if one knew $p$, one would treat $p$ in $F$.

However, given how we understand one's fully specific way of treating $p$ (i.e. as a conjunction or composite of all ways in which one treats $p$ ), this theory makes some of the same predictions as unrestricted $\forall$ AS IF. Because moving from merely believing to knowing $p$ often makes a difference to the fully specific way in which one treats $p$, as is shown by the burglar case I discussed earlier, the present theory is subject to widespread counterexample.

\subsection{Two Fully Specific Ways}

In reply, defenders of AS IF may revise their view as follows:

$\sim$ AS IF For all $x$ and $p, x$ believes $p$ iff: the fully specific way in which $x$ treats $p$ is similar to the fully specific way in which $x$ would treat $p$ if $x$ knew $p$.

The burglar case and other cases like it are no counterexamples to $\sim$ AS IF. For although the burglar treats the proposition that there is a diamond in the house (' $d$ ') differently across the case in which she merely believes and close cases in which she

\footnotetext{
22 Note that this does not mean that appeal to knowledge is per se redundant in defining belief. This is because candidate less than fully specific ways may themselves be specified by appeal to knowledge (e.g. relying on $p$ as a premise to at least the degree to which knowers rely on what they know as a premise). I thank an anonymous reviewer for this important point.

23 An anonymous reviewer suggests that defenders of the proposed regimentation of AS IF may reply that, although appeal to knowledge in clause (ii) is redundant, appeal to knowledge is not an idle wheel in coming up with the suitable domain restriction. However, this reply does not suffice for my opponents' purposes. Even if appeal to knowledge is not an idle wheel in coming up with a suitable domain restriction, this only shows that appeal to knowledge is epistemically useful in coming up with a definition of belief. Importantly, however, it does not show that knowledge features in that definition of belief. Metaphysically, then, the proposed reply does not put knowledge first, contrary to what my opponents want to do.
} 
knows, this difference does not entail that she fails to treat $d$ in similar fully specific ways across the relevant cases.

However, $\sim$ AS IF remains incomplete. Once we appeal to a similarity relation, a difficult question arises: what are the required degree(s) and respect(s) of similarity? ${ }^{24}$ We may answer this question in three ways. ${ }^{25}$

First, we may take the required degree(s) and respect(s) as primitives. But it is unclear whether our grip on these primitives suffices to know what predictions primitivist $\sim$ AS IF makes. To illustrate this, recall the burglar example. Do the fully specific way in which she treats $d$ and the fully specific way in which she would treat $d$ if she knew $d$ resemble each other in the required degree(s) and respect(s)? Given that she treats $d$ as defeasible by evidence that the diamond is under the bed, but would not do so if she knew $d$, it is natural to say, at least initially, that these two fully specific ways are dissimilar. Yet, since the burglar uses $d$ as a premise and would do so if she knew $d$, it is, on further reflection, equally natural to say that the fully specific way in which she treats $d$ is similar to the fully specific way in which she would treat $d$ if she knew it. This shows that to some degree and in some respects the two fully specific ways are not similar, whilst to some degree and in some respects they are. But which of these two matters for $\sim$ AS IF's predictions? Having said only that the required degree(s) and respect(s) of similarity are primitives, it remains unclear what predictions $\sim$ AS IF makes about the burglar.

To fill in the gaps here, we might now say that the required degree(s) and respect(s) obtain iff one believes $p$. In effect, then, we characterize them by appeal to believing $p$. This, however, makes $\sim$ AS IF circular. Some proponents of AS IF may not regard this as a problem. For instance, although Hyman (2017, p. 286) intends AS IF to be a definition of belief, he allows that his view may only provide circular, necessary and sufficient conditions for belief. ${ }^{26}$ His definition is intended to map the connections between knowing and believing $p$, rather than reducing the latter to the former. In Strawson (1992)'s evocative terms, Hyman's definition is a connective, rather than reductive, analysis. ${ }^{27}$ As a result, however, the circularity of saying that the required

\footnotetext{
24 We may deny part of the force of the question by focusing on overall similarity and suggesting that no particular respects need to be considered to settle overall similarity. However, this would still leave open what degree of similarity is relevant. Moreover, it is unclear whether comparisons of overall similarity can be made-see Morreau (2010) for discussion.

25 Strictly speaking, we may answer it in nine ways, since adopting one of the three options I sketch for degree(s) does not force one to adopt that option also for respect(s). For brevity and because the added complexity makes no difference to my argument, I proceed as if defenders of AS IF adopted one of the options for both.

26 As Roessler (2013, pp. 323-4) argues, Williamson (2000) may similarly not intend to provide noncircular, necessary and sufficient conditions for belief, regarding neither knowledge nor belief as first. However, Williamson (2020) has since explicitly formulated his view as knowledge-first. So, if circularity prevents a definition from assigning priority to belief, then Williamson now seems committed to providing a non-circular, necessary and sufficient condition for belief.

27 An anonymous reviewer raised the question whether Hyman's approach is consistent with my call for a regimentation of AS IF. I believe that it is. The case for this claim turns on the idea that regimenting AS IF remains important for doing what Hyman intends to do, namely mapping the connections between knowledge and belief. This idea is motivated by a worry one might initially have about Hyman's approach. In particular, if our view is not constrained by whether it provides non-circular necessary and sufficient conditions for belief, then how can we adjudicate between views such as to believe $p$ is to be in a state
} 
degree(s) and respect(s) of similarity obtain iff one believes $p$ does not, for Hyman, entail that $\sim$ AS IF fails as a definition of belief.

Note, however, that, if $\sim$ AS IF is circular, it does not put knowledge first. Consequently, if we are interested in a knowledge-first view of belief, Hyman's approach does not satisfy our interest. Moreover, and more substantively, it is unclear whether we can, as Hyman wants, maintain both that AS IF (or some version thereof) provides a definition of belief and that AS IF is circular. On many views of definition (e.g. Kment 2014; Rosen 2015; Horvath 2017), this possibility is ruled out. For on such views, the definiens is (in some sense) more fundamental than the definiendum. But if we appeal to believing $p$ to characterize some of the properties contained in the definiens of believing $p$ (such as the required degree(s) and respect(s) of similarity), the proposed definiens of believing $p$ is, it seems, no more fundamental than believing $p$. Of course, neither of these points are knock-down arguments against Hyman's approach. However, they highlight that it does come at a cost.

Let's turn to a second way of determining the required degree(s) and respect(s) of similarity. On this option, we let context determine the required degree(s) and respect(s). Yet this option faces many of the worries I raised earlier, even setting aside whether context successfully determines degree(s) of similarity. Partly, this is because respects of similarity are closely related to less than fully specific ways.

Since fully specific ways are conjunctions or composites of less than fully specific ways, it is natural to say that respects of similarity of such fully specific ways just are less than fully specific ways: two fully specific ways of treating $p$ are similar in a respect $F$ iff both contain $F$ as a conjunct or part. ${ }^{28}$ Introducing context-sensitivity, we may then say that two fully specific ways of treating $p$ are similar iff any/most/some contextually salient less than fully specific way contained in one is also contained in the other. ${ }^{29}$

Worries for this proposal arise from both silent and noisy contexts. To illustrate, consider a familiar noisy context. Joan does not believe that it is not raining (' $\neg r$ '). Yet she uses $\neg r$ as a premise in her meteorological computations and would do so also if she knew $\neg r$. Delia now asks whether Joan uses $\neg r$ as a premise in her computations. This makes a respect of similarity of fully specific ways of treating $\neg r$ salient: using $\neg r$ as a premise in computations. So, the fully specific way in which Joan treats

similar to knowledge', 'to believe $p$ is to resemble a knower', and Hyman's own view 'to believe $p$ is to be disposed to act, think, and feel as if one knew p'? Each view appeals to a similarity relation and, if we follow Hyman's lead, can say that the salient degree(s) and respect(s) of similarity obtain iff one believes $p$. Thus, all three views are extensionally adequate due to being circular. However, these views are not equal. Forced to choose between them, Hyman's own view seems preferable to the others. The reason for this is, I suspect, that it is more informative. Similarly, however, I take the regimentations I discuss to be more informative than unregimented AS IF. This suggests that there is reason to regiment AS IF, even on Hyman's approach.

28 Alternatively, we may say that two fully specific ways of treating $p$ are similar in a respect $F$ iff one of them contains $F$ as a conjunct or part and the other contains some conjunct or part that is similar to $F$. However, this alternative raises questions about degree(s) and extent(s) of similarity yet again. What is required for two respects (i.e. less than fully specific ways) to be similar? To what degree(s) and in what respect(s) must they resemble each other? To avoid these questions, defenders of AS IF should adopt the account in the text.

29 This is inspired by Umbach and Gust (2014)'s view of similarity. 
$\neg r$ is similar to the fully specific way in which she would treat $\neg r$ if she knew $\neg r$. Consequently, in this context, $\sim$ AS IF mistakenly predicts Joan to believe $\neg r$.

Silent and noisy contexts suggest that context is not the best tool for determining what respect(s) of similarity are required for two fully specific ways to be similar. Even setting aside whether context determines suitable degree(s) of similarity, another way of determining respect(s) of similarity is needed.

The third option for saying what degree(s) and respect(s) of similarity are required adds an explicit clause to the theory. For present purposes, I set aside how this option may work for degree(s) of similarity to focus on respect(s). Because it does not work for respect(s), it does not help defenders of $\sim$ AS IF, even if it works for degree(s).

To pursue this option, we may single out required respect(s) of similarity by singling out less than fully specific ways of treating $p$. We may say that two fully specific ways of treating $p$ are similar iff they both contain all $/ \mathrm{most} /$ some members of some, possibly singleton, set of less than fully specific ways. Candidate members of that set include using $p$ as a premise, asserting $p$, judging $p$, etc. However, as is easy to verify, this option faces the same worry as did the option of imposing a theory-driven restriction to less than fully specific ways on the domain of AS IF's quantifier. Appeal to knowledge yet again becomes an idle wheel.

\subsection{One Alternative to Similarity}

An alternative to singling out required degree(s) and respect(s) of similarity is to replace the similarity relation in $\sim$ AS IF. There are many different alternatives defenders of AS IF may propose, but one natural replacement is suggested by my burglar example. In case 1, the burglar knows that the diamond is in your house (' $d$ '). In case 2 , she believes $d$ based on the false lemma that the diamond is under the bed. Across the cases, the burglar treats $d$ in different fully specific ways. Crucially, the difference between those ways is due to the fact that, in case 2, the burglar believes $d$ based on a false lemma. Because of this, she treats $d$ as defeasible by evidence that there is no diamond under the bed.

That this difference is explained by her believing $d$ based on a false lemma, which also explains why she does not know in case 2, suggests that we identify allowed differences between fully specific ways with differences explained by the reason(s) why one does not know. More precisely, say that a fully specific way $F$ in which $x$ treats $p$ neighbours a fully specific way $G$ in which $x$ would treat $p$ if she knew $p$ iff $F$ is the same as $G$ (i.e. contains all and only the less than fully specific ways as conjuncts or parts that $G$ contains), except for differences explained by reason(s) why $x$ does not know $F$. We then amend $\sim$ AS IF accordingly:

NB AS IF For all $x$ and $p, x$ believes $p$ iff: the fully specific way in which $x$ treats $p$ neighbours the fully specific way in which $x$ would treat $p$ if she knew $p$.

NB AS IF correctly predicts that the burglar believes $d$ in case 2 of the burglar example. For her fully specific way of treating $d$ is the same as in case 1, except for differences explained by the reason why she does not know $d$, i.e. that she believes $d$ based on the false lemma that the diamond is under the bed. 
However, NB AS IF too is subject to widespread counterexample. Here is one. Kolya is sat in a chair facing a door. There is a sculpture behind the door. The door is programmed to open at $t_{2}$ and remain open until $t_{3}$, long enough for one to come to know by looking that there is a sculpture behind the door (' $s$ '). Kolya has not been told anything about what is behind the door. He has never considered $s$. Sometimes, Kolya closes his eyes or directs his attention away from the door. By chance, Kolya closes his eyes from $t_{2}$ to $t_{3}$, so does not look at the door, and for this reason does not come to know $s$.

Intuitively, Kolya does not believe $s$. And yet NB AS IF predicts Kolya to believe $s$. The reason why Kolya does not know $s$ is that he did not look at the door from $t_{2}$ to $t_{3}$. But this reason explains not only why he does not know $s$ but also the differences between his fully specific way of treating $s$ and the fully specific way in which he would treat $s$ if he knew it. If he knew $s$, he would use $s$ as a premise in his reasoning. Yet, since he did not look at the door from $t_{2}$ to $t_{3}$, he does not. If he knew $s$, he would assert $s$. Yet, since he did not look at the door from $t_{2}$ to $t_{3}$, he does not. And so on. Thus, NB AS IF mistakenly predicts Kolya to believe $s$.

Cases like Kolya's are widespread. There are many propositions about our immediate environment, which we have never considered, but which we could easily have come to know by looking. The reason why we did not come to know them was that we looked the wrong way or did not pay sufficient attention. But this reason also explains why we do not treat these propositions in the fully specific ways in which we would treat them if we knew them. So, NB AS IF predicts us to believe a wide range of propositions about our immediate environment which we have never considered. As stated then, NB AS IF must be rejected.

\section{Conclusion}

This paper developed a challenge for AS IF, the claim that to believe $p$ is to $\Phi$ as if one knew $p$. I began by arguing that $\exists$ AS IF undergenerates in silent contexts and overgenerates in noisy ones. I generalized my argument to a variety of views in $\exists$ AS IF's vicinity, which varied the type of quantifier over ways, but held fixed appeal to context to determine a domain restriction on said quantifier. I then discussed two alternative ways of regimenting AS IF, which did not appeal to context to determine a domain restriction. On the first, we imposed no domain restriction; on the second, we imposed what I called a theory-driven domain restriction. I argued that, as things stand, views of neither sort are satisfactory. In sum, we should accept none of the regimentations of AS IF I considered here. Absent a regimentation that fares better, I conclude that we should not accept AS IF.

My discussion also raises worries for extant regimentations and refinements of AS IF suggested by Williamson (2000) and Wimmer (2019). By way of conclusion, I now sketch those worries. Start with Williamson's view. In the passage from his (2000) quoted in the introduction, Williamson uses the adverbial modifier 'roughly.' The suggestion contained in that passage is an approximation only. Williamson refines it thus:

[...] to believe $p$ is to treat $p$ as if one knew $p$ - that is, to treat $p$ in ways similar to the ways in which subjects treat propositions which they know. (2000, pp.46-47) 
Evidently, this view differs from AS IF in several ways; for instance, it appeals to two proposition variables, ' $p$ ' and that introduced by 'propositions,' rather than one. Yet it also resembles one of the regimentations of AS IF I considered, viz. $\sim$ AS IF. Like $\sim$ AS IF, Williamson's refined view appeals to a similarity relation. Given this, my argument in Sect. 5.1 generalizes to Williamson's refined view. ${ }^{30}$

Wimmer (2019) suggests a refinement of AS IF according to which to believe $p$ is to $\Phi$ with respect to $p$ as one would $\Phi$ with respect to some knowable $q$ if one knew $q$. This refinement avoids several problems for AS IF outlined in Wimmer's (2019). But it does not avoid my challenge to AS IF. Although I do not have the space here to establish this claim in full generality, let me mention one problem that generalizes. Wimmer's refinement allows a proposition to be assigned to both $p$ and $q$ if it is knowable. Consequently, in the special case where $p$ is knowable, Wimmer's refinement predicts that one believes $p$ if AS IF predicts that one believes $p$. Yet this means that just as various regimentations of AS IF overgenerate, i.e. predict that one believes $p$ although one does not, so do parallel regimentations of Wimmer's refinement.

Acknowledgements For reading several earlier drafts, I am grateful to Guy Longworth. Thanks also to Tom Crowther, Richard Holton, Tristan Kreetz, Jennifer Nagel, Johannes Roessler, David Strohmaier, and Tim Williamson as well as audiences at Oxford, Pavia, and Warwick for discussion.

Funding Open Access funding enabled and organized by Projekt DEAL.

Open Access This article is licensed under a Creative Commons Attribution 4.0 International License, which permits use, sharing, adaptation, distribution and reproduction in any medium or format, as long as you give appropriate credit to the original author(s) and the source, provide a link to the Creative Commons licence, and indicate if changes were made. The images or other third party material in this article are included in the article's Creative Commons licence, unless indicated otherwise in a credit line to the material. If material is not included in the article's Creative Commons licence and your intended use is not permitted by statutory regulation or exceeds the permitted use, you will need to obtain permission directly from the copyright holder. To view a copy of this licence, visit http://creativecommons.org/licenses/by/4.0/.

\section{References}

Audi, R. N. (1994). Dispositional beliefs and dispositions to believe. Noûs, 28(4), 419-34.

Barwise, J., \& Cooper, R. (1981). Generalized quantifiers and natural language. Linguistics and Philosophy, 4(2), 159-219. https://doi.org/10.1007/BF00350139.

Bengson, J., \& Moffett, M. A. (2011). Nonpropositional intellectualism. In J. Bengson \& M. A. Moffett (Eds.), Knowing how: essays on knowledge, mind, and action (pp. 161-195). Oxford: Oxford University Press.

Boneh, N., \& Doron, E. (2010). Modal and temporal aspects of habituality*. In M. Rappaport Hovav, E. Doron, \& I. Sichel (Eds.), Lexical semantics, syntax, and event structure (pp. 338-363). Oxford: Oxford University Press. https://doi.org/10.1093/acprof:oso/9780199544325.003.0016.

Boneh, N., \& Doron, E. (2012). Hab and gen in the expression of habituality. In A. Mari, C. Beyssade, \& F. Del Prete (Eds.), Genericity (pp. 176-191). Oxford: Oxford University Press. https://doi.org/10. 1093/acprof:oso/9780199691807.003.0006.

Breckenridge, W. (2007). The meaning of look. PhD Thesis. Oxford: University of Oxford.

\footnotetext{
30 Note that, as an anonymous reviewer correctly remarks, Williamson's refined view may be amended in ways that avoid my argument. For instance, Williamson may say that to believe $p$ is to treat $p$ in the most specific way $F$ such that subjects treat propositions which they know in $F$. As this amendment does not appeal to a similarity relation, my argument in Sect. 5.1 does not generalize to this view. I plan to consider this view in future work.
} 
Buford, C., \& Cloos, C. M. (2018). A dilemma for the knowledge despite falsehood strategy. Episteme, 15(2), 166-182. https://doi.org/10.1017/epi.2016.53.

Bücking, S. (2017). Composing wie wenn: the semantics of hypothetical comparison clauses in German. Natural Language and Linguistic Theory, 35(4), 979-1025. https://doi.org/10.1007/s11049-017-93641.

van Elswyk, P. (2019). What the metasemantics of know is not. In Linguistics and Philosophy. https://doi. org/10.1007/s10988-019-09263-w.

Fara, M. (2005). Dispositions and habituals. Nous, 39(1), 43-82. https://doi.org/10.1111/j.0029-4624.2005. 00493.x.

Ferreira, M. (2016). The semantic ingredients of imperfectivity in progressives, habituals, and counterfactuals. Natural Language Semantics, 24(4), 353-397. https://doi.org/10.1007/s11050-016-91272.

Hawthorne, J., \& Manley, D. (2012). The reference book. Oxford: Oxford University Press. https://doi.org/ 10.1093/acprof:oso/9780199693672.001.0001.

Holton, R. (2017). I-facts, factives, and contrafactives. Aristotelian Society Supplementary, 91(1), $245-266$. https://doi.org/10.1093/arisup/akx003.

Horvath, J. (2017). Philosophical analysis: the concept grounding view. Philosophy and Phenomenological Research. https://doi.org/10.1111/phpr.12378.

Huddleston, R. D. \& Pullum, G. K. (2002). The Cambridge grammar of the English language. Cambridge, UK; New York: Cambridge University Press.

Hyman, J. (2017). II: knowledge and belief. Aristotelian Society Supplementary, 91(1), 267-288. https:// doi.org/10.1093/arisup/akx005.

Jaworski, W. (2009). The logic of how-questions. Synthese, 166(1), 133-155. https://doi.org/10.1007/ s11229-007-9269-3.

Kment, B. (2014). Modality and explanatory reasoning. Oxford: Oxford University Press.

Morreau, M. (2010). It simply does not add up: trouble with overall similarity. Journal of Philosophy, 107(9), 469-490. https://doi.org/10.5840/jphil2010107931.

Nagel, J. (2013). Knowledge as a Mental State. In: T. Gendler, J. Hawthorne (eds) Oxford Studies in Epistemology, vol 4. Oxford: UK: Oxford University Press, pp. 273-308.

Nagel, J. (2017). Factive and nonfactive mental state attribution. Mind and Language, 32(5), 525-544. https://doi.org/10.1111/mila.12157.

Roberts, C. (2012). Information structure in discourse: towards an integrated formal theory of pragmatics. Semantics and Pragmatics. https://doi.org/10.3765/sp.5.6.

Roessler, J. (2013). Knowledge, causal explanation, and teleology. In T. Gendler \& J. Hawthorne (Eds.), Oxford studies in epistemology 4 (pp. 321-333). Oxford: Oxford University Press.

Rosen, G. (2015). Real definition. Analytic Philosophy, 56(3), 189-209.

Schnee, I. (2015). There is no knowledge from falsehood. Episteme, 12(1), 53-74. https://doi.org/10.1017/ epi.2014.26.

Stanley, J., \& Williamson, T. (2001). Know how. The Journal of Philosophy, 98(8), 411-444.

Strawson, P. F. (1992). Analysis and metaphysics: an introduction to philosophy. Oxford; New York: Oxford University Press.

Sæbø, K. J. (2016). How questions and the manner-method distinction. Synthese, 193(10), 3169-3194. https://doi.org/10.1007/s11229-015-0924-9.

Turri, J. (2019). Knowledge from falsehood: an experimental study. Thought: A Journal of Philosophy, 8(3), 167-178. https://doi.org/10.1002/tht3.417.

Umbach, C., \& Gust, H. (2014). Similarity demonstratives. Lingua. SI: Modification at the Interfaces, 149, 74-93. https://doi.org/10.1016/j.lingua.2014.05.003.

Warfield, T. A. (2005). Knowledge from falsehood. Philosophical Perspectives, 19(1), 405-416. https:// doi.org/10.1111/j.1520-8583.2005.00067.x.

Williamson, T. (2000). Knowledge and its limits. Oxford: Oxford University Press.

Williamson, T. (2020). Knowledge, credence, and the strength of belief. Oxford

Wimmer, S. (2019). Knowledge-first believing the unknowable. Synthese. https://doi.org/10.1007/s11229019-02314-6.

Publisher's Note Springer Nature remains neutral with regard to jurisdictional claims in published maps and institutional affiliations. 